\title{
ROBÓTICA EDUCACIONAL NA EDUCAÇÃO INFANTIL: CRIAÇÃO E AVALIAÇÃO DE UMA PLATAFORMA PARA O DESENVOLVIMENTO DO PENSAMENTO COMPUTACIONAL
}

\author{
Guilherme Ballardin Duso - Exatas/UCS - gbduso@ucs.br \\ Luan Lucas Pereira de Lima - Exatas/UCS - llplima@ucs.br \\ Roberta Dall Agnese da Costa- PPGECiMa/UCS - rdacosta@ucs.br \\ Carine Geltrudes Webber - PPGECiMa/UCS - cgwebber@ucs.br
}

\begin{abstract}
Resumo. A robótica educacional vem sendo muito utilizada em escolas ao redor do mundo para o desenvolvimento de habilidades de STEM desde o começo do aprendizado. Embora existam produtos para crianças e adolescentes, há uma carência de recursos para uso na educação infantil. A pesquisa desenvolvida sustenta a importância do ensino de conceitos de programação e robótica logo nas séries iniciais. Propõe-se uma plataforma composta por hardware (robô) e software (aplicativo móvel) destinada ao ensino de programação visando o desenvolvimento de habilidades do pensamento computacional. Tal plataforma está operacional e os testes preliminares evidenciam seu potencial.
\end{abstract}

Palavras chaves: Robótica Educacional, Pensamento Computacional, Ambiente de Programação para Crianças

\section{EDUCATIONAL ROBOTICS FOR CHILDREN: AN EVALUATION OF A PLATFORM FOR THE DEVELOPMENT OF COMPUTATIONAL THINKING}

\begin{abstract}
Educational robotics has been widely used in schools around the world for development of STEM skills since the beginning of learning. The present research intends to develop a robotic platform to improve logical reasoning of school age children. Although products for children and adolescents do exist, there is a lack of resources for use in the kindergarden education. The research describe the importance of applying logic and robotics concepts on initial grades. We propose a hardware (robot) and software (app mobile) platform for programming education aiming at the development of abilities of the computational thought. Such platform is operational and the preliminary tests evidence its potential.
\end{abstract}

Keywords: Educational robotics, Computational Thinking, Children Programming Environment

\section{Introdução}

Atualmente o uso da tecnologia permeia afazeres de todas as profissões e, junto com ela, cresce a necessidade de formação de pessoas qualificadas, tanto para operar, quanto para criar tecnologias. Segundo Susilo et al. (2016) a grande demanda por profissionais nas 
áreas de STEM (STEM é um acrônimo em inglês para as disciplinas Science, Technology, Engineering and Mathematics) e a pouca procura por cursos pertencentes a elas, tem levado alguns países a incentivar, de forma prioritária, o acesso de graduandos a esses campos de atuação. Ainda assim, tradicionalmente cursos destas áreas tem baixa procura (SUSILO et al., 2016).

Para estes profissionais, o raciocínio lógico e o pensamento computacional são habilidades fundamentais e estão ligadas a racionalidade do pensamento, a resolução de problemas e a utilização de abstrações de técnicas da ciência da computação no cotidiano (WING, 2006). A compreensão do conceito e das aplicações do pensamento computacional nos insere em um contexto de ensino amplo, em que é possível utilizar múltiplos níveis de abstração para resolver os mais diversos tipos de problemas.

A partir dos estudos de Wing (2006) percebeu-se a importância e a necessidade de acrescentar os conceitos de resolução de problemas e pensamento computacional nos currículos escolares, além de promover reflexões sobre quais ferramentas podem ser utilizadas para incentivar e validar o desenvolvimento destas habilidades. Essas ferramentas educacionais, conforme evidenciado por Lin (2015), ainda são pouco estudadas no âmbito da aprendizagem, para o desenvolvimento das habilidades de STEM em escolas primárias. Deste modo, segundo Boucinha et al. (2017) o pensamento computacional tem sido comparado com a alfabetização do século XXI, pois possuir habilidades básicas de codificação se tornou condição necessária para muitos postos de trabalho.

Ramos et al. (2015) confirmam que ainda faltam interessados nas áreas de STEM durante o ensino secundário e também na Universidade, revelando uma grande preocupação em desenvolver o interesse por elas. Resnick et al. (2009) explicam que, objetivo de inserir habilidades destas áreas no currículo básico das escolas perpassa o desenvolvimento de estratégias para criar, utilizar a lógica, desenvolver soluções e ter a habilidade de discernir entre as possibilidades oferecidas, e não simplesmente utilizar as ferramentas tecnológicas como entretenimento.

Documentos oficiais que normatizam a Educação no Brasil já sinalizam em seus textos-base uma abordagem relativa a construção de conhecimentos, competências, atitudes e valores, voltados, por exemplo, à utilização de conhecimentos e da linguagem digital para entender e explicar a realidade (BRASIL, 2016). Estas aprendizagens específicas, dizem respeito às práticas de linguagem em ambiente digital, que têm modificado as práticas de linguagem em diferentes campos de atuação social, relacionando-se, portanto, com as habilidades de STEM. Corroborando com estas colocações, Kologeski et al. (2016) destacam que, como a tecnologia está presente no cotidiano das pessoas, ela deve ser inserida nas práticas de ensino.

Apesar disso, ainda se observam dificuldades para inserção da lógica computacional em todos os níveis de ensino (Resnick et al., 2009). Esta constatação motiva a busca por alternativas eficientes para o aprendizado destas habilidades alinhadas a conteúdos tradicionalmente escolares. Uma das alternativas para isso é a utilização da robótica educacional como contributo para o professor no ensino (SILVA et al., 2016; AGUIAR et al., 2015; FERREIRA et al., 2016; RICHTER et al., 2016) que enfatiza a importância da utilização do computador como intermediador do ensino e da aprendizagem (MAISONNETTE, 2002).

Assim, diante do exposto, colocam-se os objetivos desta pesquisa: 0 desenvolvimento de um componente de hardware programável e de fácil manuseio para 
ser utilizado no contexto escolar brasileiro; a criação de um aplicativo que promova situações de interação e desafio para desenvolver o raciocínio lógico de crianças da Educação Infantil e a testagem e avaliação das ferramentas desenvolvidas. Assim, a plataforma de ensino e aprendizagem proposta pela presente pesquisa integra hardware e software, que compõem um ambiente atrativo para os estudantes, visando proporcionar o desenvolvimento da lógica e raciocínio, apoiando-se nas proposições de Chin, Hong e Chen (2014) e Chaudhary et al. (2016).

\section{Material e Métodos}

A pesquisa foi desenvolvida em três etapas: etapa I - cujo objetivo foi desenvolver um componente de hardware programável e de fácil manuseio para ser utilizado no contexto escolar brasileiro; etapa II - criar um aplicativo que promova situações de interação e desafio para desenvolver o raciocínio lógico de crianças da Educação Infantil; etapa III - testagem e avaliação das ferramentas desenvolvidas.

Trata-se, portanto, de uma investigação de cunho qualitativo. A abordagem escolhida para este trabalho foi a pesquisa-ação. Sendo assim, de acordo com ZuberSkerritt (2004), todo projeto ou programa de pesquisa-ação tem dois objetivos: (1) melhoria da prática, inovação, mudança ou desenvolvimento de práticas sociais e (2) melhor entendimento por parte dos participantes de suas práticas. A pesquisa-ação não está comprometida em resolver problemas, no sentido de tentar descobrir o que está errado, mas sim em buscar conhecimento sobre como podemos melhorar ou mudar a nossa prática (FERRANCE, 2000).

Deste modo, a figura 1, a seguir, ilustra a arquitetura de software do aplicativo responsável pelo controle, gerenciamento dos estudantes e propostas de desafios (a esquerda) e o componente de hardware da forma que será disposto na carroceria (a direita).

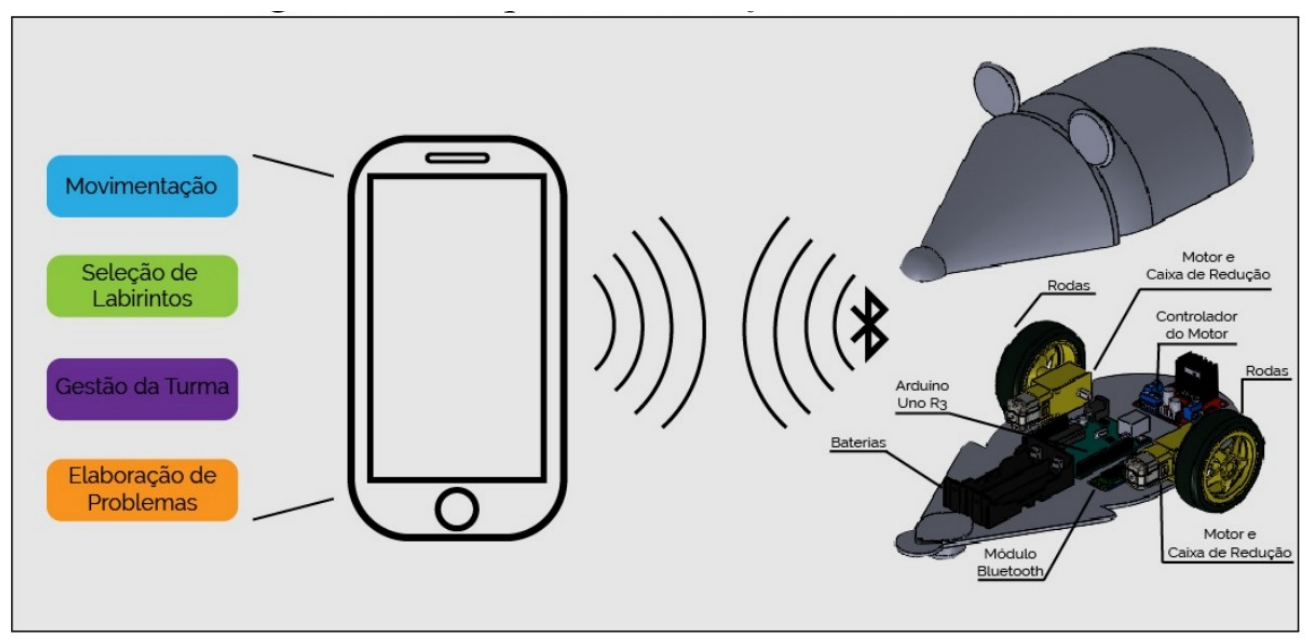

Figura 1 - Arquitetura de software e componente de hardware.

$\mathrm{Na}$ etapa I, para o desenvolvimento do robô computacional, foi escolhido o microprocessador Arduino. De origem italiana, o Arduino possui um código aberto e 
tem sido utilizado em muitos projetos amadores e profissionais, por seu hardware robusto e por sua interface intuitiva. Além disso, com a proposta de tornar o componente de hardware uma plataforma com possibilidade de movimentação de acordo com as necessidades do usuário, foi aplicado ao robô dois motores com rodas integradas. O kit utilizado possui um motor DC com caixa de redução acoplada e rodas com dimensional exato para o acoplamento com a caixa de redução. Para controlar o motor deve ser utilizado um controlador de motor, para que a corrente exigida não fosse maior que a permitida pelas saídas do microcontrolador e para a possibilidade de acionamento nas duas direções. Além disso, o componente desenvolvido tinha como pré-requisito a possibilidade de o usuário movimentar o componente de hardware de forma a desenvolver a lógica, para isso foi aplicada a comunicação por meio do protocolo Bluetooth.

Após a realização de uma revisão de ferramentas semelhantes disponíveis observou-se a importância de se desenvolver um produto robusto e com um design atraente para os estudantes da educação infantil. Para tanto, a carroceria foi planejada no software de modelagem 3D SolidWorks e impressa por meio da tecnologia 3D.

Em função do público-alvo desta pesquisa tratar-se de participantes da educação infantil, por razões de segurança, houve a necessidade de um planejamento ainda mais rigoroso da carroceria em relação a robustez, não deixando qualquer componente frágil do produto exposto. Também foram observadas características como a facilidade de montagem e, preferencialmente, que as partes que envolvem a carroceria fossem encaixáveis. Isto tanto para evitar o uso de parafusos expostos, quanto para facilitar a montagem do equipamento.

$\mathrm{Na}$ etapa II, optou-se pelo desenvolvimento de um aplicativo para o sistema operacional móvel Android. A escolha por este sistema ocorreu, principalmente, por ser uma plataforma móvel e gratuita. O aplicativo foi implementado em linguagem de programação Java. O aplicativo utiliza a comunicação Bluetooth com um robô desenvolvido em Arduino.

Assim, dentro da proposta apresentada, o ambiente de ensino e aprendizagem tem como componentes o mediador, que pode ser o próprio professor, e os estudantes. O mediador tem a incumbência de elaborar os desafios que serão solucionados pelos estudantes, além da inserção deles, através do cadastro da turma junto ao software e o gerenciamento, o controle do componente de hardware.

A proposta desta investigação disponibilizou, além do professor de cada turma, um monitor com formação específica em tecnologias. Isto porque, segundo Abellón (2015), o fato de a maioria dos docentes da área da Pedagogia ou mesmo nas licenciaturas, recebem pouco ou nenhum referencial sobre a utilização da tecnologia na Educação em seus cursos de formação inicial.

Após a elaboração das propostas dos problemas (desafios), os estudantes interagiram com o aplicativo de controle executando atividades de programação do robô, que movimentava-se de forma que o desafio proposto pelo mediador fosse solucionado. Ainda considerando as movimentações disponíveis no software, há possibilidade de criar laços de repetição, que facilitam o desenvolvimento da lógica nos estudantes, compondo novas possibilidades de solução dos problemas.

O aplicativo, portanto, foi projetado, para a utilização por dois sujeitos: o mediador, que tem acesso total a ele; os estudantes que somente tem disponível a 
resolução dos cenários. Assim, o software exibe a execução dos cenários, além de transmitir os comandos que o estudante definiu para serem executados no robô.

A avaliação do produto se deu de acordo com as reações dos estudantes. $\mathrm{O}$ estudo observou a motivação deles no interesse demonstrado e pelo entusiasmo em resolver os problemas propostos. Outros pontos que foram avaliados são o desempenho do produto frente a lógica desenvolvida pelos alunos, a robustez do produto em se manter funcional durante toda interação em sala de aula e a intuitividade, revelando-se de fácil operação.

Para a coleta de dados foi utilizado o SQLite, isto porque, o Android por padrão, tem suporte completo à biblioteca para criação e acesso ao banco de dados SQLite. Essa familiarização do Android com o SQLite foi um fator fundamental para a utilização desta ferramenta. A biblioteca SQLite, é um gerenciador de banco de dados independente de servidor e sem necessidade de configurações, leve e seguro (COSTA; SANTOS, 2015).

\section{Resultados e discussão}

\subsection{Os cenários de ensino}

Cenários de ensino, este nome foi dado aos desafios propostos pelo mediador e desenvolvidos no aplicativo pelos estudantes. Cada cenário é composto por um tabuleiro, representado por $n$ linhas e $m$ colunas. O ponto de início é representado por um ratinho e o fim pelo queijo. Podem existir k queijos em um cenário, e ele só é concluído após o ratinho comer todos os queijos. Pode-se ainda inserir os obstáculos. A figura 2 ilustra três cenários de utilização. As informações de cada cenário são armazenadas em tabelas e, com isso, é possível a inserção, edição e exclusão de cenários pelo professor.

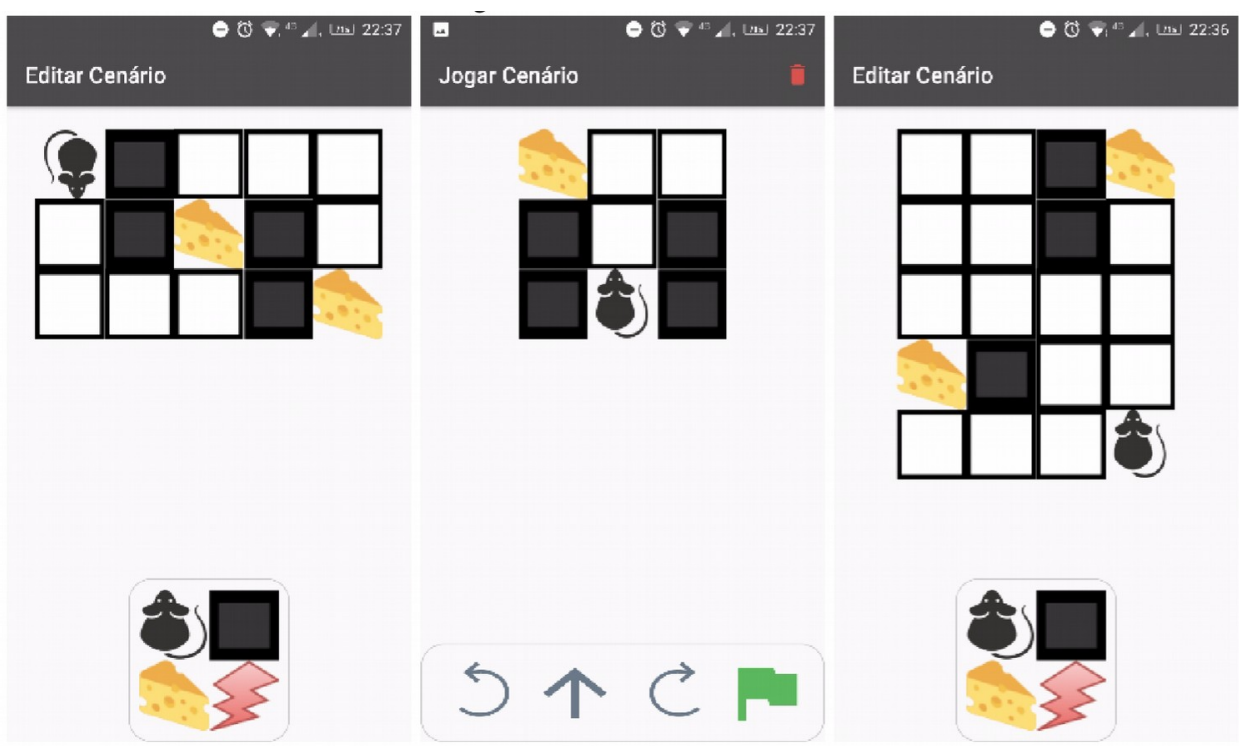

Figura 2 - Três diferentes cenários de ensino. 
Os cenários são, portanto, o cerne do trabalho. É possível criar cenários de ensino de qualquer tamanho e as dificuldades são definidas pelo professor. Após a definição do tamanho do cenário, é preciso definir na matriz qual será o ponto de partida e o ponto de término do cenário. Pode-se, adicionalmente, inverter a orientação do robô e com isso estimular o estudante a executar os comandos espelhados, tornando a complexidade maior.

\subsection{Resolução dos cenários}

Após clicar na bandeira verde, um laço é iniciado e os comandos são enviados para o robô via Bluetooth, um a um. Na tela do aplicativo, é ilustrado o movimento do robô. Existem quatro possibilidades de resultado, após o estudante clicar na bandeira verde: a primeira é o robô cair em um buraco (obstáculo em preto na matriz); a segunda é sair do cenário; a terceira é movimentar-se pelo cenário e não concluir o objetivo; a quarta é comer todos os queijos e concluir o cenário com sucesso. Após a execução do cenário, os comandos que foram utilizados pelos estudantes, são armazenados na tabela 'CenariosAluno'. Com isso, é possível fazer as consultas dos resultados dos cenários.

\subsection{Testagem e avaliação}

A etapa de testagem e avaliação foi organizada em três intervenções didáticas. $\mathrm{Na}$ primeira delas, os estudantes foram familiarizados com noções básicas de lógica aplicada a resolução de problemas. Para tanto, eles deveriam resolver os cenários de maneira física, ou seja, eles eram os atores e deveriam mover-se, segundo as orientações dos colegas, até o objetivo final. Na segunda intervenção didática, os estudantes foram apresentados ao robô e ao aplicativo, trabalhando com noções de movimentos no aplicativo, movimentos do robô e resolução de situações-problema. Para tanto, foram propostas as tarefas I e II (descritas a seguir). Já na terceira intervenção didática, eles deveriam também resolver os cenários de utilização com o robô. Para tanto, foi proposta a tarefa III e reaplicada a tarefa II.

Participaram desta pesquisa dezoito estudantes (sendo cinco do Pré e treze do Jardim), em uma escola privada de educação infantil da região sul do Brasil. A segunda e a terceira intervenção didática tiveram duração de quatro horas cada. Foram analisados aspectos como a interação dos estudantes com a proposta e o tempo de resolução dos cenários. Em todas as intervenções utilizou-se a estratégia de trabalho em grupos, contendo dois ou três estudantes cada.

Logo no início já foi percebida a empolgação dos estudantes com a proposta. Da mesma forma, Cambruzzi e Souza (2015), em sua pesquisa com o objetivo comparar o ensino de lógica computacional utilizando a robótica, e os meios convencionais de ensino, a avaliação do estudante evidencia a preferência dos alunos pela utilização da robótica no ensino.

Após este primeiro contato, foi apresentado o primeiro cenário de utilização (Tarefa I, figura 3 (a)). Um cenário simples em que os estudantes interagiram por um tempo relativamente curto para atingir os objetivos propostos pelo professor.

Para a Tarefa II (figura 3 (a)) foi utilizado um cenário que propunha maior dificuldade. As dificuldades consistiam em: o desafio de pegar dois queijos; e o desafio da compreensão da necessidade de movimento do ratinho para posicionar-se frente ao queijo. Ambos cenários foram resolvidos pelos estudantes, porém no segundo cenário foi necessária uma maior medição do professor pelo fator da maior dificuldade. Nesse 
cenário, devido ao aumento da dificuldade em função da abstração, os estudantes também demonstraram mais dificuldades na resolução.

Com terceira intervenção didática (Tarefa III), foi proposto um cenário ainda mais complexo. A dificuldade atrelada a este cenário (Figura 3 (b)) estava na compreensão da necessidade de movimentação do ratinho e, para isso, a execução de comandos espelhados. Foi sugerido aos estudantes que se moverem pelo cenário físico, a fim conseguirem resolver de maneira real o que foi proposto. Nesse cenário, em função do aumento da abstração, observaram-se maiores dificuldades para a solução do problema.
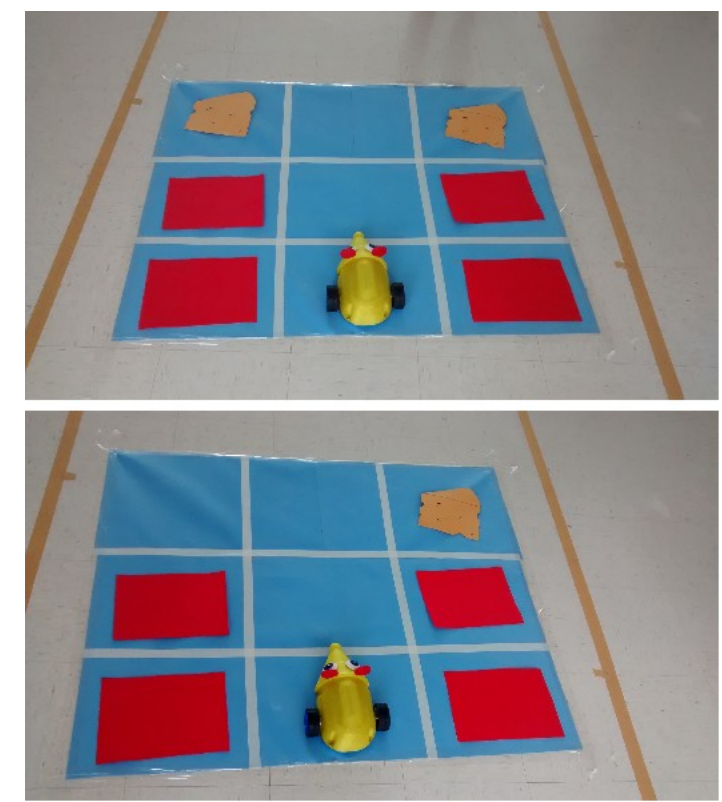

(a)

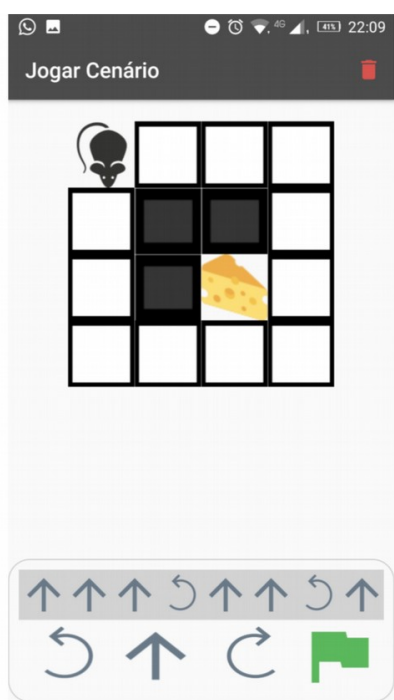

(b)

Figura 3 - Cenário de ensino das Tarefas I ((a) tabuleiro inferior), Tarefa II ((a) tabuleiro superior) e Tarefa III ((b) tela do aplicativo)

Em seguida, planejou-se uma reaplicação da Tarefa II. E, como era de se esperar, os estudantes demonstraram menos dificuldades na resolução, resolvendo-a, inclusive, em menor tempo.

Por se tratar de uma pesquisa-ação, que, segundo definições de Ferrance (2002) é um processo que segue um ciclo em que se aprimora a prática pela oscilação sistemática entre o agir no campo da prática e a investigação a respeito dela, na terceira intervenção didática foram realizadas algumas melhorias, em relação a segunda. Foi observado, por exemplo, a necessidade de acrescentar imagens relacionadas as ações do ratinho robô. Assim foram inseridas imagens de um ratinho caindo e de um ratinho pensativo. Estes acréscimos surgiram a partir da observação das falas dos estudantes, questionando o por que não acontecia nada quando ele caia em um buraco. 
Outra alteração realizada foi na comunicação das ações do robô. Observou-se que não estava sendo enviado ao robô o comando que o fizesse se chocar com a parede ou com os obstáculos. Com isso, o robô parava antes de executar o comando que faria isto acontecer. Assim, os estudantes não conseguiam entender o que de fato estava ocorrendo, que o comando estava errado, pois o robô simplesmente parava. Diante desta constatação, foram adicionados sons característicos de batida, para facilitar a identificação do erro de comando. Além deste, também foi acrescentado ao robô o comando que fazia com que ele se movesse para o obstáculo ou para fora do cenário, com o objetivo de tornar visível aos estudantes que um erro de comando aconteceu.

Com a consulta dos dados (Tabela 1) foi possível analisar o tempo de realização de cada uma delas.

Tabela 1 - Tempo de realização das tarefas pelos alunos das turmas de pré e jardim.

\begin{tabular}{|c|c|c|c|c|}
\hline \multirow{2}{*}{ Acompanhamento } & \multicolumn{2}{|c}{$18 / 10 / 17$} & \multicolumn{2}{c|}{ 25/10/17 } \\
\hline Aluno & Tarefa 1 & Tarefa 2 & Tarefa 3 & Tarefa 2 \\
\hline 1-Pré & $00: 01: 11$ & $00: 00: 38$ & $00: 05: 36$ & $00: 02: 23$ \\
2-Pré & $00: 02: 05$ & $00: 04: 30$ & $00: 03: 33$ & $00: 02: 13$ \\
\hline 3-Pré & $00: 00: 53$ & $00: 00: 42$ & $00: 05: 44$ & $00: 00: 41$ \\
\hline 4-Pré & $00: 01: 02$ & $00: 02: 47$ & $00: 02: 25$ & $00: 01: 04$ \\
\hline 5-Pré & $00: 02: 19$ & $00: 00: 38$ & $00: 06: 11$ & $00: 04: 47$ \\
\hline 1-Jardim & $00: 01: 14$ & $00: 03: 31$ & $00: 04: 03$ & $00: 01: 21$ \\
\hline 2-Jardim & $00: 01: 02$ & $00: 03: 19$ & $00: 03: 35$ & $00: 02: 19$ \\
\hline 3-Jardim & $00: 01: 12$ & $00: 04: 04$ & $00: 03: 35$ & $00: 02: 19$ \\
\hline 4-Jardim & $00: 01: 20$ & $00: 01: 19$ & $00: 03: 35$ & $00: 02: 19$ \\
\hline 5-Jardim & $00: 01: 03$ & $00: 02: 45$ & $00: 03: 03$ & $00: 01: 00$ \\
\hline 6-Jardim & $00: 01: 39$ & $00: 01: 52$ & $00: 04: 03$ & $00: 04: 47$ \\
\hline 7-Jardim & $00: 00: 55$ & $00: 05: 40$ & $00: 02: 18$ & $00: 02: 06$ \\
8-Jardim & $00: 01: 49$ & $00: 02: 07$ & $00: 02: 10$ & $00: 01: 41$ \\
\hline 9-Jardim & $00: 01: 06$ & $00: 02: 19$ & $00: 02: 10$ & $00: 01: 41$ \\
\hline 10-Jardim & $00: 02: 07$ & $00: 02: 50$ & $00: 02: 10$ & $00: 01: 41$ \\
\hline 11-Jardim & $00: 06: 14$ & $00: 06: 16$ & não realizou & não realizou \\
\hline 12-Jardim & $00: 04: 00$ & $00: 06: 16$ & $00: 02: 00$ & $00: 01: 08$ \\
\hline 13-Jardim & $00: 04: 00$ & $00: 06: 16$ & $00: 01: 50$ & $00: 01: 30$ \\
\hline
\end{tabular}

Assim, pode-se concluir que pré e jardim não tiveram grandes diferenças em seus tempos, apesar da diferença de um ano de idade, em média. Além disso, observouse uma importante melhora na redução do tempo de execução da Tarefa II quando ela foi reaplicada. Já com a execução da Tarefa III foi observada a possibilidade de aumentar a dificuldade de execução do cenário de ensino apenas alterando a orientação do ratinho.

\section{Considerações finais}

O aplicativo elaborado tem a função de dar suporte ao professor, com cenários de ensino adaptáveis a diferentes contextos, idades e conhecimentos e também com a possibilidade de consultar os dados da evolução dos alunos nos cenários de utilização. 
Os cenários de ensino, portanto, podem ser desafios que simulam um jogo, com o objetivo de motivar os estudantes a partir do desenvolvimento de competências voltadas à resolução de problemas, típica do pensamento computacional. E, por fim, observou-se que a utilização de robótica educacional pode mobilizar aspectos lúdicos e concretos, com o objetivo de aprimorar as estratégias de ensino e aprendizagem para a Educação Infantil.

Assim, com esta pesquisa pretendeu-se desenvolver uma alternativa para inserção da lógica de programação e de habilidades do pensamento computacional para crianças. Além disso, cabe destacar que existe uma proposta de tornar o aplicativo um produto educacional, agregando novas funcionalidades através de novos testes. Acredita-se que, deste modo, o conhecimento produzido na academia possa ser útil e significativo também fora dela.

\section{Referências}

ABELLÓN, M. Professor: as dificuldades para utilizar a tecnologia dentro da sala de aula dasescolas públicas brasileiras. Disponível em: <http://direcionalescolas.com.br/2015/08/06/ professor-as-dificuldades-para-utilizar-atecnologia-dentro-da-sala-de-aula-das-escolaspublicas-brasileiras/>. Acesso em: 17 junho2017.

AGUIAR, Y. de et al. Introdução à robótica e estímulo à lógica de programação no ensino básico utilizando o kit educativo Lego, Simpósio Brasileiro de Informática na Educação (SBIE), 2015. Anais... [S.I.: s.n.], 2015. p. 1418-1424.

BOUCINHA, R.M.; BRACKMANN, C.P.; BARONE, D.A.C.; CASALI, A. Construção do pensamento computacional através do desenvolvimento de games. RENOTE, v. 15, n. 1, 2017.

CAMBRUZZI, E.; SOUZA, R. M. de. Robótica educativa na aprendizagem de lógica deprogramacção: aplicação e análise. In: XXI WORKSHOP DE INFORMÁTICA NA ESCOLA(WIE 2015), 2015. Anais. . [S.I.: s.n.], 2015.

CHAUDHARY, $V$. et al. An experience report on teaching programming and computational thinking to elementary level children using lego robotics education kit. In: IEEE EIGHTH INTERNATIONAL CONFERENCE ON TECHNOLOGY FOR EDUCATION (T4E), 2016., 2016. Anais. . . [S.I.: s.n.], 2016. p. 38-41.

CHIN, K. Y.; HONG, Z. W.; CHEN, Y. L. Impact of using an educational robot-based learning system on students 2019; motivation in elementary education. IEEE Transactions on Learning Technologies, [S.I.], v. 7, n. 4, p. 333-345, Oct 2014.

COSTA, D. P.; SANTOS, M. T. P. Comparativo entre gerenciadores de banco de dados paraaplicação android. Revista TIS, São Carlos, p. 20-30, 2015.

FERRANCE, E. (2000). Action research: LAB, Northeast and Island Regional Education Laboratory at Brown University.

FERREIRA, L. A. C. et al. Se-robô: aplicativo para robótica educacional de baixo custo. In:SIMPÓSIO BRASILEIRO DE INFORMÁTICA NA EDUCAÇÃO, 2016, Passo Fundo. Anais. . . [S.I.: S.n.], 2016. p. 846-855.

KOLOGESKI, A.L.; SILVA, C.G.; BARBOSA, D.N.F.; MATTOS, R.R.; MIORELLI, ST. Desenvolvendo o Raciocínio Lógico e o Pensamento Computacional: Experiências no Contexto do Projeto Logicando. RENOTE, v. 14, n. 2, 2016.

LIN, V. J. Computational thinking and technology toys. Honors Thesis Collection, [S.I.], v. 307, 2015. 
MAISONNETTE, R. A utilização dos recursos informatizados a partir de uma relação inventiva com a máquina: a robótica educativa. In: PROINFO - PROGRAMA NACIONAL DE INFORMÁTICA NA EDUCAÇÃO, 2002, Paraná. Anais. . . [S.I.: s.n.], 2002.

MINISTÉRIO DA EDUCAÇÃO. Diretrizes curriculares nacionais para a educaçãoinfantil. Brasilia, 2010. Disponível em:<http://ndi.ufsc.br/files/2012/02/Diretrizes-Curriculares-para-a-EI.pdf>. Acesso em: 17 jun. 2017.

RAMOS, E. et al. Pensamento computacional na escola e práticas de avaliação das aprendizagens. uma revisão sistemática da literatura. Challenges 2015: Meio século de TIC na Educação, Half a century of ICT in Education, [S.I.], p. 595-846, 2015.

RESNICK, M. et al. Scratch: programming for all. Communications of the ACM, [S.I.],v. 52, n. 11, p. 60-67, 2009.

RICHTER, D. R. et al. Design de um brinquedo programável para crianças de 4 a 5 anos deidade através da metodologia design thinking. In: ESCOLA REGIONAL DE INFORMÁTICA DA SOCIEDADE BRASILEIRA DE COMPUTAÇÃO (SBC), 2016, Itajaí. Anais. . . [S.I.: s.n.], 2016.

SANTOS, C. B. et al. Robotics and programming: attracting girls to technology. In: INTERNATIONAL CONFERENCE ON ADVANCES IN COMPUTING, COMMUNICATIONS AND INFORMATICS (ICACCI), 2016., 2016. Anais. . [S.I.: s.n.], 2016. p. 2052-2056.

SILVA, D. P. da et al. Aplicação de robótica na educação de forma gradual para o estímulo do pensamento computacional. In: WORKSHOPS DO CONGRESSO BRASILEIRO DE INFORMÁTICA NA EDUCAÇÃO, 2016, Minas Gerais. Anais. . . [S.I.: s.n.], 2016.

SUSILO, E. et al. Stormlab for stem education: an affordable modular robotic kit for integrated science, technology, engineering, and math education. IEEE Robotics Automation Magazine, [S.I.], v. 23, n. 2, p. 47-55, June 2016.

WING, J. M. Computational thinking. Communications of the ACM, [S.I.], v. 49, n. 3,p. 33-35, 2006.

ZUBER-SKERRITT, Val Roche O., (2004) A constructivist model for evaluating postgraduate supervision: a case study, Quality Assurance in Education, Vol. 12 Issue: 2, pp.82-93. 